\title{
Automorphic Forms on the Expanded Symmetric Domain of Type IV
}

\author{
By
}

\author{
Hiroki AOKI*
}

\begin{abstract}
We introduce a lifting from a given Jacobi form of index 1 to an automorphic form on an expanded domain of type $\mathrm{IV}$, introduced by Saito [20, 22]. The method is a generalization of Gritsenko $[10,11]$ for symmetric domain of type $\mathbb{N}$. We constract a lifting function satisfying a certain translation formula on the expanded domain.
\end{abstract}

$\S 0$. Introduction

$\S 1$. Expanded symmetric domain of type IV

$\S 2$. Expanded Jacobi forms

$\S 3$. Lifting function of Jacobi forms

$\S 4$. Arithmetic lifting on the expanded domain

\section{§0. Introduction}

It is classically known as the Jacobi's inversion problem to find a description of the coordination of a Riemann surface in terms of integrals on the Riemann surface. In case of an elliptic curve, this was solved by the use of elliptic modular functions. In general for higher genus case, this was solved by the use of theta functions (cf. Siegel [23]). One may consider similar problem for the integrals on higher dimensional varieties. In this case, the main problem is to construct automorphic forms on the domain of periods. The case for family of polarized $K 3$ surfaces was studied by Pyatetski-Shapiro [18], where the domain of periods is the classical symmetric domain of type IV. Even in this case, we do not have a full description of the ring of automorphic forms.

Communicated by K. Saito, April 17, 1998. Revised October 16, 1998

1991 Mathematics Subject Classifications: 11F55, 14J17

* Graduate School of Research Institute for Mathematical Sciences, Kyoto University, Kyoto 606-8502, Japan.

e-mail: hiroki@kurims.kyoto-u.ac.jp 
Saito [19] introduced another theory of period integrals of primitive forms for any universal unfolding of a singularity, where the period domain is no more the classical domain. On the other hand, the inversion problem for this case gets a precise structure, because of some particular generator system, called the flat generator system on the theory of primitive forms. Except for a few cases, called simple singularities and simply elliptic singularities (cf. Satake [24]), there is almost no answer known on the flat inversion problem.

The next nontrivial case is the 14 exceptional unimodular singularities, that is introduced by Arnold [2]. The unfolding theory of these singularities was studied by several authors (cf. Brieskorn [5], Dolgacev-Nikulin [6], Looijenga $[13,14]$ and Pinkham [17]), where there is 1-codimensional subspace of the deformation space which is identified with the deformation space of certain polarized $K 3$ surfaces. Hence the period domain should be a 1-dimensional expansion of the symmetric domain of type $\mathrm{V}$. Looijenga [13] suggested the expansion in connection with the triangle singularity. In fact, both domains studied by Saito and Looijenga coincide for these cases. Hence it is a natural problem to find flat generator system on the ring of automorphic forms on the expanded domain, where the weights of the generators are prescribed. The present article is to contribute for the construction of nontrivial automorphic forms on the expanded domain.

The construction of nontrivial automorphic forms on the classical domain of type IV was studied by several authors (cf. Oda [15], Zagier [26], Sugano [25], Borcherds [4] and Gritsenko [10,11]). Following the idea of Gritsenko [11], in present article, we construct automorphic forms on the expanded domain by lifting a Jacobi form of index 1 .

In section 1, we define the expanded domain and the group action on it, according to Saito $[19,20]$.

In section 2 , we define and survey a Jacobi form on the expanded domain.

In section 3 , we construct a function $f_{m}: \mathbb{C} \times \mathbb{H} \rightarrow \mathbb{C}(m \in \mathbb{N})$, which has the translation formula

$$
f_{m}(t, \tau)=\exp \left(-\pi \sqrt{-1} \frac{m c t}{c \tau+d}\right) f_{m}\left(\frac{t}{(c \tau+d)^{2}}, \frac{a \tau+b}{c \tau+d}\right)
$$

for any $\left(\begin{array}{ll}a & b \\ c & d\end{array}\right) \in S L_{2}(\mathbb{Z})$. By multiplying $f_{m}$, a Jacobi form of index $m$ on the classical domain is lifted to a Jacobi form of index $m$ on the expanded domain.

In section 4, we construct a formal power series with an automorphic property, from a Jacobi form of index 1 on the expanded domain. In case of a lifted Jacobi form, the formal power series gives a holomorphic automorphic form on the expanded domain. 


\section{§1. Expanded Symmetric Domain of Type N}

Let $L$ be an even integral lattice of rank $l+4$, with an inner product defined by a quadratic form $S$ of signature $(2, l+2)$, where $l \in \mathbb{N}=\{1,2,3, \cdots\}$, and $V:=$ $L \otimes_{\mathbb{Z}} \mathbb{R}$ be a base vector space of $L$. We suppose the case $L=L_{1} \oplus I I_{1,1}$ and $L_{1}=L_{0}$ $\bigoplus I I_{1,1}$, where $I I_{1,1}$ is a hyperbolic plane. We fix one coordinate, such that

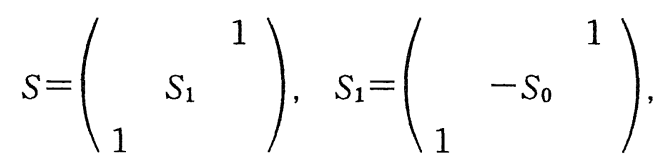

where $S_{0}$ is an even integral positive definite matrix. We denote $A[B]:={ }^{t} B A B$ and $A\{B\}:={ }^{t} \bar{B} A B$ for matrixes $A, B$. Let $X^{+}$be one of the connected component of $X$, where $X$ is a topological space. If $X$ is a topological group, we choose $X^{+}$ with the identity element.

The classical symmetric domain of type $\mathrm{N}$ is defined by

$$
D_{V}:=\left\{V^{\prime} \subset V \mid \operatorname{dim}_{\mathbb{R}} V^{\prime}=l+2, V^{\prime}<0\right\},
$$

where $V^{\prime}<0$ means $\left.S\right|_{V^{\prime}}<0$. We define a left action of $g \in G:=O^{+}(S, \mathbb{R})$ on $D_{V}$ by $V^{\prime} \mapsto g V^{\prime}$. We fix one orthogonal decomposition of $V=V_{+} \oplus V_{-}$such that $V_{+}>$ 0 and $V_{-}<0$, and let $K:=S O\left(V_{+}\right) \times S O\left(V_{-}\right)$be subgroup of $G$. Then the group $G$ acts on $D_{V}$ transitively and the group $K$ is the stabilizer of $V_{-}$. Hence $D_{V}$ is isomorphic to $G / K$ as a real manifold, and $D_{V}$ is a homogeneous domain.

We define a complex domain

$$
H:=\left\{z \in L \bigotimes_{\mathbb{Z}} \mathbb{C} \mid S\{z\}>0, S[z]=0\right\},
$$

and let $\mathrm{P}_{\mathbb{C}} H$ be the $\mathbb{C}$-projective space of $H$. We remark that $H$ and $\mathrm{P}_{\mathbb{C}} H$ have two connected components. Then the domain $D_{V}$ is isomorphic to $\left(\mathrm{P}_{\mathbb{C}} H\right)^{+}$as a real manifold. The induced action of $G$ on $\left(\mathrm{P}_{\mathbb{C}} H\right)^{+}$is $z \mapsto g z\left(g \in G, z \in\left(\mathrm{P}_{\mathbb{C}} H\right)^{+}\right)$. This action preserves the complex structure of $\left(\mathrm{P}_{\mathbb{C}} H\right)^{+}$. We define a complex domain

$$
\mathscr{H}_{S}:=\left\{w={ }^{t}\left(w_{1}, w_{2}, \cdots, w_{l+2}\right) \in L_{1} \bigotimes_{\mathbb{Z}} \mathbb{C} \mid S_{1}[\operatorname{Im}(w)]>0\right\}
$$

which has two connected components. The domain $\mathscr{H}_{S}^{+}$is isomorphic to $\left(\mathrm{P}_{\mathbb{C}} H\right)^{+}$ as a complex manifold. For simplicity, we choose the connected component of $\mathscr{H}_{S}^{+}$by $\operatorname{Im}\left(w_{1}\right)>0$. The induced action of $g=\left(g_{i, j}\right)_{0 \leq i, j \leq l+3} \in G$ on $\mathscr{H}_{S}^{+}$, induced by the action on $\left(\mathrm{P}_{\mathbb{C}} H\right)^{+}$is

$$
w \mapsto g(w)=\left(\frac{-\frac{1}{2} g_{i, 0} S_{1}[w]+\sum_{j=1}^{l+2} g_{i, j} w_{j}+g_{i, l+3}}{j(g, w)}\right)_{1 \leq i \leq l+2}
$$


where the denominator

$$
j(g, w):=-\frac{1}{2} g_{l+3,0} S_{1}[w]+\sum_{j=1}^{l+2} g_{l+3, j} w_{j}+g_{l+3, l+3}
$$

becomes an automorphic factor. This action preserves the complex structure of $\mathscr{H}_{S}^{+}$. For a holomorphic function $F$ on $\mathscr{H}_{S}^{+}, k \in \mathbb{Z}$, and $g \in G$, we define $\left.F\right|_{k} g$ by $\left(\left.F\right|_{k} g\right)(w):=j(g, w)^{-k} F(g(w))$. This gives a right action of $G$ on the set of all holomorphic functions on $\mathscr{H}_{S}^{+}$. Let $\Gamma$ be a discrete subgroup of $G$. The action of $\Gamma$ on $\mathscr{H}_{S}^{+}$is properly discontinuous. A holomorphic function $F$ on $\mathscr{H}_{S}^{+}$is called an automorphic form of weight $k$ on $\mathscr{H}_{S}^{+}$with respect to the group $\Gamma$ if $F$ satisfies the equation $\left.F\right|_{k} \Gamma=F$. The set of all automorphic forms of weight $k$ on $\mathscr{H}_{S}^{+}$with respect to $\Gamma$ is a $\mathbb{C}$-vector space and written as $\mathbb{M}_{k}\left(\mathscr{H}_{S}^{+}, \Gamma\right)$.

The expanded domain introduced by Saito [20] is defined by

$$
B_{V}:=\left\{\varphi \in \operatorname{Hom}_{\mathbb{R}}(V, \mathbb{C}) \mid \operatorname{Ker}(\varphi)<0\right\},
$$

which $G$ acts by $\varphi \mapsto \varphi \circ g^{-1}$. However, this action is not transitive on $B_{V}^{+}$. To make the action transitive. we consider another action. We define a left action of $G L_{2}^{+}(\mathbb{R})$ on $\mathbb{C}$ by $\left(\begin{array}{l}\operatorname{Re}(A z) \\ \operatorname{Im}(A z)\end{array}\right):=\left(\begin{array}{ll}a & b \\ c & d\end{array}\right)\left(\begin{array}{l}\operatorname{Re}(z) \\ \operatorname{Im}(z)\end{array}\right)$, where $A=\left(\begin{array}{ll}a & b \\ c & d\end{array}\right) \in G L_{2}^{+}(\mathbb{R})$.

Hence we can consider the left action of $G \times G L_{2}^{+}(\mathbb{R})$ on $B_{V}^{+}$by $\varphi \mapsto A \circ \varphi \circ g^{-1}$. This action is transitive. Now, we fix one $\mathbb{R}$-isomorphism $\iota: V_{+} \rightarrow \mathbb{C}$ and define $\tilde{\imath}$ $\in B_{V}^{+}$by $\tau_{V_{+}}=\iota$ and $\tau_{V_{-}}=0$. Let $\Delta S O\left(V_{+}\right):=\left\{(g, \widetilde{g}) \mid g \in S O\left(V_{+}\right), \widetilde{g}=\iota \circ g \circ \iota^{-1}\right\}$, $\Delta S O\left(V_{-}\right):=\left\{\left(g, E_{2}\right) \mid g \in S O\left(V_{-}\right)\right\}$, and $\tilde{K}:=\Delta S O\left(V_{+}\right) \times \Delta S O\left(V_{-}\right)$be subgroups of $G \times G L_{2}^{+}(\mathbb{R})$. We can easily show that the group $\widetilde{K}$ is the stabilizer of $\widetilde{\tau} \in B_{V}^{+}$. Hence $B_{V}^{+}$is isomorphic to $\left(G \times G L_{2}^{+}(\mathbb{R})\right) / \widetilde{K}$ as a real manifold, and $B_{V}^{+}$is the homogeneous domain.

The domain, considered by Looijenga [13], is

$$
B:=\left\{z \in L \bigotimes_{\mathbb{Z}} \mathbb{C}|S\{z\}-| S[z] \mid>0\right\},
$$

and let $\mathrm{P}_{\mathbb{C}} B$ be the $\mathbb{C}$-projective space of $B$. We remark that $B$ and $\mathrm{P}_{\mathbb{C}} B$ have two connected components. Saito [20] showed that $B_{V}$ is isomorphic to $B$ as a complex manifold. The induced action of $G \times G L_{2}^{+}(\mathbb{R})$ on $B^{+}$is given by $z \mapsto g z$ and $\left(\begin{array}{l}\operatorname{Re}(z) \\ \operatorname{Im}(z)\end{array}\right) \mapsto\left(\begin{array}{ll}a & b \\ c & d\end{array}\right)\left(\begin{array}{c}\operatorname{Re}(z) \\ \operatorname{Im}(z)\end{array}\right)$. Of course, this action is transitive, but it does not preserve the complex structure of $B^{+}$. The subgroup whose action preserve the complex structure is $G \times \mathbb{C}^{\times}$, where we recognize $\mathbb{C}^{\times} \hookrightarrow G L_{2}^{+}(\mathbb{R})$ by a $+b \sqrt{-1}$ $\mapsto\left(\begin{array}{cc}a & b \\ -b & a\end{array}\right)$. By the restriction to this subgroup, Saito [20] induced the action 
of $G$ on $\left(\mathrm{P}_{\mathbb{C}} B\right)^{+}$. The induced action is $[z] \mapsto[g z]$, which preserves the complex structure of $\left(\mathrm{P}_{\mathbb{C}} B\right)^{+}$. This action is the expansion of the action of $G$ on $\left(\mathrm{P}_{\mathbb{C}} H\right)^{+}$. hence not transitive. But we remark that the value inv $(z):=\frac{|S[z]|}{S\{z\}}(0 \leq \operatorname{inv}(z)$ $<1)$ is invariant with respect to the action of $G$.

Now, we realizer this expanded domain $\left(\mathrm{P}_{\mathbb{C} B}\right)^{+}$as a subset of complex Euclidean space. Let

$$
\mathscr{B}_{S}:=\left\{Z=\left(\begin{array}{c}
t \\
w
\end{array}\right) \in \mathbb{C} \oplus\left(L_{1} \otimes_{\mathbb{Z}} \mathbb{C}\right) \mid S_{1}[\operatorname{Im}(w)]>\frac{|t|-\operatorname{Re}(t)}{2}\right\},
$$

which has two connected components. The correspondence

$$
\mathscr{B}_{S} \ni\left(\begin{array}{c}
t \\
w
\end{array}\right) \leftrightarrow\left[\left(\begin{array}{c}
\frac{t-S_{1}[w]}{2} \\
w \\
1
\end{array}\right)\right] \in \mathrm{P}_{\mathbb{C} B}
$$

gives the isomorphism $\mathscr{B}_{S} \cong \mathrm{P}_{\mathbf{C}} B$ as a complex manifolds. For simplicity, we choose the connected component of $\mathscr{B}_{S}^{+}$by $\operatorname{Im}\left(w_{1}\right)>0$, where $w={ }^{t}\left(w_{1}, \cdots, w_{l+2}\right)$. We remark

$$
\operatorname{inv}(Z)=\frac{|t|}{\operatorname{Re}(t)+2 S_{1}[\operatorname{Im}(w)]}
$$

By an easy calculation, we can write the induced action of $G$ on $\mathscr{B}_{S}^{+}$as follows,

$$
Z \mapsto g(Z)=\left(\begin{array}{c}
\frac{t}{J(g, Z)^{2}} \\
\left(\frac{\frac{1}{2} g_{i, 0}\left(t-S_{1}[w]\right)+\sum_{j=1}^{l+2} g_{\imath, j} w_{j}+g_{\imath, l+3}}{J(g, Z)}\right)_{1 \leq_{\imath} \leq l+2}
\end{array}\right),
$$

where the denominator

$$
J(g, Z):=\frac{1}{2} g_{l+3,0}\left(t-S_{1}\left[w^{\prime}\right]\right)+\sum_{j=1}^{l+2} g_{l+3, j} w_{j}+g_{l+3, l+3}
$$

becomes an automorphic factor. For a holomorphic function $F$ on $\mathscr{B}_{S}^{+}, k \in \mathbb{Z}$, and $g \in G$, we define $\left.F\right|_{k} g$ by $\left(\left.F\right|_{k} g\right)(Z):=J(g, Z)^{-k} F(g(Z))$. This gives a right action of $G$ on the set of all holomorphic functions on $\mathscr{B}_{S}^{+}$. The action of $\Gamma$ on $\mathscr{B}_{S}^{+}$ is properly discontinuous. A holomorphic function $F$ on $\mathscr{B}_{S}^{+}$is called an automorphic form of weight $k$ on $\mathscr{B}_{S}^{+}$with respect to the group $\Gamma$ if $F$ satisfies $\left.F\right|_{k} \Gamma=F$. The set of all automorphic forms of weight $k$ on $\mathscr{B}_{S}^{+}$with respect to $\Gamma$ is a 
$\mathbb{C}$-vector space and written as $\mathbb{M}_{k}\left(\mathscr{B}_{S}^{+}, \Gamma\right)$. We remark $t \in \mathbb{M}_{-2}\left(\mathscr{B}_{S}^{+}, \Gamma\right)$ for any $\Gamma$. The following diagram means the relation of the spaces in this article.

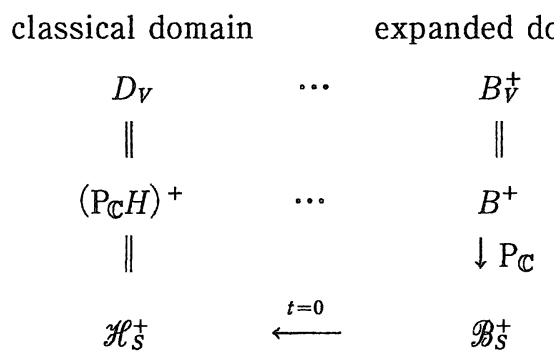

Remark. The classical symmetric domain with respect to the quadratic form $\widetilde{S}:=S \oplus(-2)$

$$
\mathscr{H}_{\tilde{S}}:=\left\{\left(\begin{array}{c}
w \\
t
\end{array}\right) \mid S_{1}[\operatorname{Im}(w)]-2(\operatorname{Im}(t))^{2}>0\right\}
$$

is the double covering space of the expanded symmetric domain $\mathscr{B}_{S}^{+}$by

$$
\pi: \mathscr{H}_{\tilde{S}}^{+} \ni\left(\begin{array}{c}
w \\
t
\end{array}\right) \mapsto\left(\begin{array}{c}
2 t^{2} \\
w
\end{array}\right) \in \mathscr{B}_{S}^{+}
$$

Let $\sigma:\left(\begin{array}{c}w \\ t\end{array}\right) \mapsto\left(\begin{array}{c}w \\ -t\end{array}\right)$ be an involutive isomorphism of $\mathscr{H}_{\tilde{s}}^{+}$. Then we have the induced isomorphism

$$
\text { c: } \mathscr{H}_{\bar{S}}^{+} /\langle\sigma\rangle \simeq \mathscr{B}_{S}^{+}
$$

We define a $G$-action on $\mathscr{H}_{\tilde{S}}^{ \pm}$by $e: G=O^{+}(S, \mathbb{R}) \hookrightarrow O^{+}(\widetilde{S}, \mathbb{R})$. It follows that $\pi$ and $c$ are equivariant with the group action of $G$. Hence for any finite index subgroup $\widetilde{\Gamma} \subset G L(l+5, \mathbb{Z}) \cap O^{+}(\widetilde{S}, \mathbb{R})$, we have an injective map

$$
\widetilde{\tau}: \mathbb{M}_{k}\left(\mathscr{H}_{S}^{+}, \widetilde{\Gamma}\right)^{\sigma} \longleftrightarrow \mathbb{M}_{k}\left(\mathscr{B}_{S}^{+}, \Gamma\right),
$$

where $\Gamma:=e^{-1}\left(\widetilde{\Gamma} \cap e\left(O^{+}(S, \mathbb{R})\right)\right)$. The image of $\widetilde{\tau}$ is the finite dimensional $\mathbb{C}$-vector space. But indeed, $\mathbb{M}_{k}\left(\mathscr{B}_{S}^{+}, \Gamma\right)$ is not finite dimensional. In this article, we construct automorphic forms on $\mathscr{B}_{S}^{+}$, not via $\mathscr{H}_{\tilde{S}}^{+}$. The relation between automorphic forms in this article and the image of $\tau$ has not been clear.

\section{§2. Expanded Jacobi Forms}

From now on, we fix the discrete subgroup $\Gamma:=G \cap O(S, \mathbb{Z})$. For simplicity, we write $w={ }^{t}\left(\omega,{ }^{t} \xi, \tau\right)$, where $\omega=w_{1}, \xi={ }^{t}\left(w_{2}, \cdots, w_{l+1}\right)$ and $\tau=w_{l+2}$. To 
investigate automorphic forms and Jacobi forms, we define the following matrixes as elements of $G$,

$$
\begin{aligned}
& {[A]:=\left(\begin{array}{ccc}
A^{*} & & \\
& E_{l} & \\
& & A
\end{array}\right)} \\
& \left(A=\left(\begin{array}{ll}
a & b \\
c & d
\end{array}\right), A^{*}:=\frac{1}{a d-b c}\left(\begin{array}{cc}
a & -b \\
-c & d
\end{array}\right)\right) \text {, } \\
& {[U]:=\left(\begin{array}{lll}
E_{2} & & \\
& U & \\
& & E_{2}
\end{array}\right)} \\
& \left(U \in S O\left(S_{0}, \mathbb{R}\right)\right) \text {, } \\
& {[x, y]:=\left(\begin{array}{ccccc}
1 & 0 & { }^{t} y S_{0} & 0 & \frac{1}{2} S_{0}[y] \\
0 & 1 & { }^{t} x S_{0} & \frac{1}{2} S_{0}[x] & { }^{t} x S_{0} y \\
0 & 0 & E_{l} & x & y \\
0 & 0 & 0 & 1 & 0 \\
0 & 0 & 0 & 0 & 1
\end{array}\right)} \\
& {[r]:=\left(\begin{array}{ccccc}
1 & 0 & 0 & -r & 0 \\
0 & 1 & 0 & 0 & r \\
0 & 0 & E_{l} & 0 & 0 \\
0 & 0 & 0 & 1 & 0 \\
0 & 0 & 0 & 0 & 1
\end{array}\right)} \\
& (r \in \mathbb{R}) \text {, }
\end{aligned}
$$

Using these symbols, we can write the parabolic groups and a Jacobi group as follows,

$$
\begin{aligned}
& \mathrm{P}_{\mathbb{R}}^{+}=\left\langle\left[G L_{2}^{+}(\mathbb{R})\right],\left[S O\left(S_{0}, \mathbb{R}\right)\right],\left[\mathbb{R}^{l}, \mathbb{R}^{l}\right],[\mathbb{R}]\right\rangle, \\
& \mathrm{P}_{\mathbb{R}}^{\prime}=\left\langle\left[S L_{2}(\mathbb{R})\right],\left[S O\left(S_{0}, \mathbb{R}\right)\right],\left[\mathbb{R}^{l}, \mathbb{R}^{l}\right],[\mathbb{R}]\right\rangle, \\
& \mathrm{P}_{\mathbb{Z}}^{+}=\mathrm{P}_{\mathbb{R}}^{+} \cap \Gamma=\left\langle\left[S L_{2}(\mathbb{Z})\right],\left[S O\left(S_{0}, \mathbb{Z}\right)\right],\left[\mathbb{Z}^{l}, \mathbb{Z}^{l}\right],[\mathbb{Z}]\right\rangle .
\end{aligned}
$$

For any $k, m \in \mathbb{Z}$, we can define an action of $\mathrm{P}_{\mathbb{R}}^{J}$ on $\left\{\varphi: \mathbb{C} \times \mathbb{C}^{l} \times \mathbb{H} \rightarrow \mathbb{C}\right\}$ as follows,

$$
\begin{aligned}
& \left(\left.\varphi\right|_{k, m}[A]\right)(t, \xi, \tau)=(c \tau+d)^{-k} \mathbf{e}\left(\frac{-m c\left(t+S_{0}[\xi]\right)}{2(c \tau+d)}\right) \varphi\left(\frac{t}{(c \tau+d)^{2}}, \frac{\xi}{c \tau+d} \frac{a \tau+b}{c \tau+d}\right), \\
& \left(\left.\varphi\right|_{k, m}[U]\right)(t, \xi, \tau)=\varphi(t, U \xi, \tau) \\
& \left(\left.\varphi\right|_{k, m}[x, y]\right)(t, \xi, \tau)=\mathbf{e}\left(m\left({ }^{t} x S_{0} \xi+\frac{1}{2} S_{0}[x] \tau+{ }^{t} x S_{0} y\right)\right) \varphi(t, \xi+x \tau+y, \tau)
\end{aligned}
$$




$$
\left(\left.\varphi\right|_{k, m}[r]\right)(t, \xi, \tau)=\mathbf{e}(m r) \varphi(t, \xi, \tau),
$$

where $\mathbb{e}(z):=\exp (2 \pi \sqrt{-1} z)$.

A holomorphic function $\varphi$ is called an expanded Jacobi form of weight $k$ and index $m$ with respect to the group $\mathrm{P}_{\mathbb{Z}}^{+}$if $\varphi$ satisfies the equation $\left.\varphi\right|_{k, m} \mathrm{P}_{\mathbb{Z}}^{+}=\varphi$ and if $\varphi$ has the Fourier expansion

$$
\varphi(t, \xi, \tau)=\sum_{\substack{n \in \mathbb{N} \in\left\{0,0, u \in L_{0}^{\prime} \\ 2 n m \geq \geq 0\right.}} A_{(n, u)}(t) \mathbb{e}\left(-{ }^{t} u S_{0} \xi+n \tau\right),
$$

where $L_{0}^{\prime}$ is the dual lattice with respect to $L_{0}$. The set of all expanded Jacobi forms of weight $k$ and index $m$ with respect to the group $\mathrm{P}_{\mathbb{Z}}^{+}$is a $\mathbb{C}$-vector space and written as $\mathbb{J}_{k, m}\left(\mathscr{B}_{S}^{+}, \mathrm{P}_{\mathbb{Z}}^{+}\right)$. Of couse, restricting this definition within $t=0$, we have the definition of $\mathbb{J}_{k, m}\left(\mathscr{H}_{S}^{+}, \mathrm{P}_{\mathbb{Z}}^{+}\right)$.

Lemma 2.1. Let

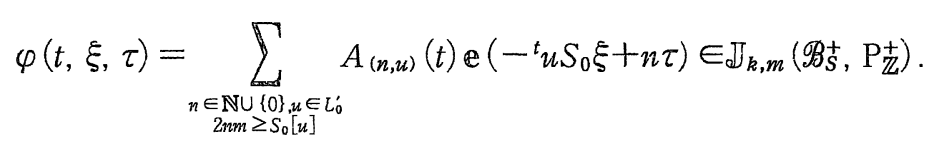

The function $A_{(n, u)}$ only depends on the values $u \bmod m L_{0}$ and $2 n m-S_{0}[u]$.

Proof. It is obvious from the action of $[x, 0]$, where $x \in \mathbb{Z}^{l}$.

The first important matter is to show that any Fourier coefficient of an automorphic form on $\mathscr{B}_{S}^{+}$with respect to $\omega$ is a Jacobi form.

Lemma 2.2. Let $p \in \mathbb{N}$ and $F$ be a holomorphic function on $\mathscr{B}_{S}^{+}$. If $F$ is $\left[(p \mathbb{Z})^{l},(p \mathbb{Z})^{l}\right]$-invariant, we have a Fourier expansion

$$
F(Z)=\sum_{m=0}^{\infty} \varphi_{m}(t, \xi, \tau) \mathbf{e}(m \omega)
$$

and $\varphi_{0}(t, \xi, \tau)$ does not depend on $\xi$.

Proof. This proof is similar to the one of the book of Eichler-Zagier [8, Theorem 1.2].

Corollary 2.3。 If $m<0$, then $\mathbb{J}_{k, m}\left(\mathscr{B}_{S}^{+}, \mathrm{P}_{\mathbb{Z}}^{+}\right)=0$.

Proof. It is obvious from Lemma 2.2.

Proposition 2.4. If $F(Z) \in \mathbb{M}_{k}\left(\mathscr{B}_{S}^{+}, \Gamma\right)$, we have the Fourier expansion 


$$
\begin{aligned}
F(Z) & =\sum_{m=0}^{\infty} \varphi_{m}(t, \xi, \tau) \mathbf{e}(m \omega) \\
& =\sum_{\lambda={ }^{t}\left(n,{ }^{t} u^{t}, m\right) \in \Lambda} \psi_{\lambda}(t) \mathbf{e}\left({ }^{t} \lambda S_{1} w\right),
\end{aligned}
$$

where $\Lambda:=\left\{\lambda={ }^{t}\left(n,{ }^{t} u, m\right) \in L_{1}^{\prime} \mid n \geq 0, m \geq 0, S_{1}[\lambda] \geq 0\right\}$. Hence $\varphi_{m} \in \mathbb{J}_{k, m}\left(\mathscr{B}_{S}^{+}, \mathrm{P}_{\mathbb{Z}}^{+}\right)$.

Proof. Let $F(Z)=\sum_{\lambda \in L_{1}^{\prime}} \psi_{\lambda}(t)$ e $\left.{ }^{(t} \lambda S_{1} w\right)$. By Lemma 2.2 and $(\omega \leftrightarrow \tau)-$ invariance of $F$, it is enough to show that $\phi_{\lambda}(t)=0$ if $S_{1}[\lambda]<0$ and $n, m>0$. We assume that there exists a triple $\lambda^{\prime}=(\alpha, \beta, \gamma)$ such that $\phi_{\lambda^{\prime}}(t) \neq 0, \alpha, \gamma>0$ and $S_{1}\left[\lambda^{\prime}\right]<0$. To show the contradiction, we put

$$
P:=\left(\begin{array}{ccc}
1 & 0 & 0 \\
-v & E_{l} & 0 \\
\frac{1}{2} S_{0}[v] & { }^{t} v S_{0} & 1
\end{array}\right) \text { and } \widetilde{P}:=\left(\begin{array}{ccc}
1 & 0 & 0 \\
0 & P & 0 \\
0 & 0 & 1
\end{array}\right) \in G,
$$

where $v:=-\frac{\beta}{\alpha}$. The function

$$
\widetilde{F}(Z):=\left.F\right|_{k} \widetilde{P}^{-1}(Z)=F\left(\begin{array}{c}
t \\
P^{-1} w
\end{array}\right)
$$

is $\widetilde{P} \Gamma \widetilde{P}^{-1}$-invariant. By a direct calculation of $\widetilde{P}^{-1}[x, y] \widetilde{P}$, we have $\left[\left(\alpha^{2} \mathbb{Z}\right)^{l}\right.$, $\left.\left(\alpha^{2} \mathbb{Z}\right)^{l}\right] \subset \widetilde{P} \Gamma \widetilde{P}^{-1}$. Hence $\widetilde{F}$ is $\left[\left(\alpha^{2} \mathbb{Z}\right)^{l},\left(\alpha^{2} \mathbb{Z}\right)^{l}\right]$-invariant. By a similar calculation, we know that $\widetilde{F}$ has some integer period for any component of $w$. Therefore we have the Fourier expansion

$$
\begin{aligned}
\tilde{F}\left(\begin{array}{c}
t \\
w
\end{array}\right) & \left.=\sum_{\lambda \in L_{1}^{\prime}} \phi_{\lambda}(t) \mathbf{e}^{(t} \lambda S_{1} P^{-1} w\right) \\
& \left.=\sum_{\lambda \in L_{1}^{\prime}} \phi_{\lambda}(t) \mathbf{e}^{t}(P \lambda) S_{1} w\right) \\
& =\sum_{\lambda \in P L_{1}^{\prime}} \phi_{P^{-1} \lambda}(t) \mathbf{e}\left(\lambda S_{1} w\right)
\end{aligned}
$$

Therefore, by the assumption, $\phi_{\lambda^{\prime}}(t)$, the Fourier coefficient of the term $\mathbf{e}\left(P \lambda^{\prime} S_{1} w\right)$ of $\widetilde{F}$, is not zero. However, $\widetilde{F}$ is $\left\{[x, y] \mid x, y \in\left(\alpha^{2} \mathbb{Z}\right)^{l}\right\}$-invariant. Hence from the Lemma 2.2 the Fourier coefficient of the term $\mathbf{e}\left(P \lambda^{\prime} S_{1} w\right)$ is zero. This completes the proof.

Fact 2.5. Let $\varphi \in \mathbb{J}_{k, m}\left(\mathscr{B}_{S}^{+}, \mathrm{P}_{\mathbb{Z}}^{+}\right)$and $\lambda, \mu \in \mathbb{Q}^{l}$. Then there exists some group 
$\Gamma^{\prime}$, which is a finite index subgroup of $S L_{2}(\mathbb{Z})$, such that

$$
\phi(\tau):=\mathbf{e}\left(\frac{m}{2} S_{0}[\lambda] \tau\right) \varphi(0, \lambda \tau+\mu, \tau)
$$

is a modular form of weight $k$ with respect to $\Gamma^{\prime}$. This $\Gamma^{\prime}$ depends only on $\lambda$ and $\mu$. Hence have the following results.

(1) If $m=0$ and $k<0$, then $\left.\varphi\right|_{t=0}=0$ for any $\varphi \in \mathbb{J}_{k, 0}\left(\mathscr{B}_{S}^{+}, \mathrm{P}_{\mathbb{Z}}^{+}\right)$.

(2) If $m=0$ and $k=0$, then $\left.\varphi\right|_{t=0}$ is a constant function for any $\varphi \in \mathbb{J}_{0,0}\left(\mathscr{B}_{S}^{+}, \mathrm{P}_{\mathbb{Z}}^{+}\right)$.

(3) If $m>0$ and $k \leq 0$, then $\left.\varphi\right|_{t=0}$ is a constant function for any $\varphi \in \mathbb{J}_{k, m}\left(\mathscr{B}_{S}^{+}, \mathrm{P}_{\mathbb{Z}}^{+}\right)$.

It is easily induced by Eichler-Zagier [8, Theorem 1.3].

Corollary 2.6. We have the following results.

(1) If $k<0$ and $F \in \mathbb{M}_{k}\left(\mathscr{B}_{S}^{+}, \Gamma\right)$, then $\left.F\right|_{t=0}=0$. Hence $t^{-1} F \in \mathbb{M}_{k+2}\left(\mathscr{B}_{S}^{+}, \Gamma\right)$.

(2) If $k=0$ and $F \in \mathbb{M}_{0}\left(\mathscr{B}_{S}^{+}, \Gamma\right)$, then $\left.F\right|_{t=0}$ is a constant function. Hence there exists some $c \in \mathbb{C}$ such that $t^{-1}(F-c) \in \mathbb{M}_{2}\left(\mathscr{B}_{S}^{+}, \Gamma\right)$.

(3) If $k<0$, then $\mathbb{M}_{k}\left(\mathscr{H}_{s}^{+}, \Gamma\right)=0$. If $k=0$, then $\mathbb{M}_{0}\left(\mathscr{H}_{s}^{+}, \Gamma\right)=\mathbb{C}$.

Proof. It is obvious from previous propositions.

Proposition 2.7. If $l$ is even and $k$ is odd, then $\mathbb{M}_{k}\left(\mathscr{B}_{S}^{+}, \Gamma\right)=\{0\}$, and $\mathbb{M}_{k}\left(\mathscr{H}_{S}^{+}, \Gamma\right)=\{0\}$.

Proof. If $l$ is even, then $-E_{l+4} \in \Gamma$. Hence $F=(-1)^{k} F$ for any $F \in \mathbb{M}_{k}\left(\mathscr{B}_{S}^{+}\right.$, $\Gamma)$ and $F \in \mathbb{M}_{k}\left(\mathscr{H}_{S}^{+}, \Gamma\right)$.

Let

$$
A\left(\mathscr{H}_{S}^{+}, \Gamma\right):=\bigoplus_{k=-\infty}^{\infty} \mathbb{M}_{k}\left(\mathscr{H}_{S}^{+}, \Gamma\right)=\bigoplus_{k=0}^{\infty} \mathbb{M}_{k}\left(\mathscr{H}_{S}^{+}, \Gamma\right)
$$

and

$$
A\left(\mathscr{B}_{S}^{+}, \Gamma\right):=\bigoplus_{k=-\infty}^{\infty} \mathbb{M}_{k}\left(\mathscr{B}_{S}^{+}, \Gamma\right)
$$

be graded rings.

Theorem 2.8. Automorphic froms of positive weight and $t$ generate $A\left(\mathscr{B}_{S}^{+}, \Gamma\right)$ over $\mathbb{C}$. If $l$ is even, automorphic forms of positive even weight and $t$ generate $A\left(\mathscr{B}_{S}^{+}\right.$, $\Gamma)$ over $\mathbb{C}$.

Proof. It is obvious from Corollary 2.6 and Proposition 2.7. 
Theorem 2.9. There exists a subring $R$ of $A\left(\mathscr{H}_{S}^{+}, \Gamma\right)$, such that

$$
A\left(\mathscr{B}_{S}^{+}, \Gamma\right) / t A\left(\mathscr{B}_{S}^{+}, \Gamma\right) \cong R .
$$

Proof. It is obvious from the homomorphism

$$
p:\left.A\left(\mathscr{B}_{S}^{+}, \Gamma\right) \ni F \mapsto F\right|_{t=0} \in A\left(\mathscr{H}_{S}^{+}, \Gamma\right) .
$$

\section{§3. Lifting Function of Jacobi Forms}

In this section, we construct a function $f_{m}$, which gives the map

$$
\mathbb{J}_{k, m}\left(\mathscr{H}_{S}^{+}, \mathrm{P}_{\mathbf{Z}}^{+}\right) \stackrel{\times f_{m}}{\longrightarrow} \mathbb{J}_{k, m}\left(\mathscr{B}_{S}^{+}, \mathrm{P}_{\mathbb{Z}}^{+}\right)
$$

The following fact is called Cauchy's estimate.

Fact 3.1. Let $f(z)$ be a holomorphic function on $\{|z-a| \leq r\}$ and $|f(z)| \leq M$. Then we have

$$
\left|f^{(n)}(a)\right| \leq M n ! r^{-n}
$$

The proof is, for example, in the book of Ahlfors [1, p.122].

For any $m \in \mathbb{Z}$, let

$$
f_{m, j}(\tau):= \begin{cases}\frac{2(m \pi \sqrt{-1})^{j}}{j !(j-1) !}\left(\frac{d^{j}}{d \tau^{j}} \log \eta(\tau)\right) & (j \in \mathbb{N}) \\ 1 & (j=0)\end{cases}
$$

and

$$
f_{m}(t, \tau):=\sum_{j=0}^{\infty} f_{m, j}(\tau) t^{i}
$$

where $\eta(\tau)$ is the Dedekind's eta function

$$
\eta(\tau):=\mathbf{e}\left(\frac{\tau}{24}\right) \prod_{n=1}^{\infty}(1-\mathbf{e}(n \tau)) \quad(\tau \in \mathbb{H}) .
$$

We remark

$$
f_{m, j+1}(\tau)=\frac{m \pi \sqrt{-1}}{j(j+1)} f_{m, j}^{\prime}(\tau)
$$

for any $j \in \mathbb{N}$. 
Proposition 3.2. The function $f_{m}$ is holomorphic on $\mathbb{C} \times \mathbb{H}$.

Proof. Let $a \in \mathbb{H}, 0<r<\frac{\operatorname{Im}(a)}{2}$, and $M:=\max _{|\tau-a| \leq 2 r}|\log \eta(\tau)|$. By Fact 3.1, we have

$$
\left|\frac{d^{j}}{d \tau^{j}} \log \eta(\tau)\right| \leq M j ! r^{-j} \quad(|\tau-a| \leq r)
$$

Hence the series (2) converges absoluteiy and locally uniformly on $\mathbb{C} \times \mathbb{H}$, by Hadamard's formula for the radius of convergence.

Proposition 3.3. We have the Fourier expansion

$$
f_{m}(t, \tau)=1-\frac{m \pi^{2}}{6} t+\sum_{n=1}^{\infty}\left(\sum_{j=1}^{\infty} \frac{(-2)^{j+1} m^{j} \pi^{2 j} n^{j-1} \sigma_{1}(n)}{j !(j-1) !} t^{j}\right) \mathbb{e}(n \tau)
$$

where $\sigma_{k}(n)=\sum_{a \mid n} a^{k}$.

Proof. It is obvious from the following $q$-expansion

$$
\frac{d}{d \tau} \log \eta(\tau)=\frac{\pi \sqrt{-1}}{12}-2 \pi \sqrt{-1} \sum_{n=1}^{\infty} \sigma_{1}(n) q^{n}
$$

where $q:=e(\tau)$.

Lemma 3.4. We have the translation formula

$$
f_{m, j}(\tau)=\sum_{n=0}^{j} \frac{(-m c \pi \sqrt{-1})^{j-n}}{(j-n) !(c \tau+d)^{j+n}} f_{m, n}\left(\frac{a \tau+b}{c \tau+d}\right)
$$

for any $\left(\begin{array}{ll}a & b \\ c & d\end{array}\right) \in S L_{2}(\mathbb{Z})$.

Proof. The case $j=0$, the equation (6) is obvious. The case $j=1$, we have the equation (6) from the equation $\frac{\eta^{\prime}}{\eta}(\tau)=(c \tau+d)-2 \frac{\eta^{\prime}}{\eta}\left(\frac{a \tau+b}{c \tau+d}\right)-\frac{c}{2(c \tau+d)}$. The case $j \geq 2$, we use the induction on $j$. We have the equation (6) by the assumption of the induction and the equation (3).

Theorem 3.5. Let $\varphi \in \mathbb{J}_{k, m}\left(\mathscr{H}_{S}^{+}, \mathrm{P}_{\mathbb{Z}}^{+}\right)$be a Jacobi form on $\mathscr{H}_{S}^{+}$and $\widetilde{\varphi}(t, \xi$, $\tau):=\varphi(\xi, \tau) f_{m}(t, \tau)$ be a holomorphic function on $\mathscr{B}_{S}^{+}$. Then $\widetilde{\varphi} \in \mathbb{J}_{k, m}\left(\mathscr{B}_{S}^{+}, \mathrm{P}_{\mathbb{Z}}^{+}\right)$and $\left.\widetilde{\varphi}\right|_{t=0}=\varphi$. 
Proof. By Proposition 3.2 and Proposition 3.3, it is enough to show the translation formula

$$
f_{m}(t, \tau)=\mathbf{e}\left(\frac{-m c t}{2(c \tau+d)}\right) f_{m}\left(\frac{t}{(c \tau+d)^{2}}, \frac{a \tau+b}{c \tau+d}\right)
$$

for any $\left(\begin{array}{ll}a & b \\ c & d\end{array}\right) \in S L_{2}(\mathbb{Z})$. By a direct calculation, we have this translation formula from Lemma 3.4 .

\section{§4. Arithmetic Lifting on the Expanded Domain}

In this section, we construct an automorphic form from an expanded Jacobi form of index 1 . About the case of the classical domain of type $\mathbb{N}$, see Gritsenko [11].

Let $\mathrm{P}_{\mathbb{Q}}^{+}:=\mathrm{P}_{\mathbb{R}}^{+} \cap G L_{l+4}(\mathbb{Q})$. We define an action of the Hecke ring $\mathrm{H}\left(\mathrm{P}_{\mathbb{Z}}^{+}, \mathrm{P}_{\mathbb{Q}}^{+}\right)$ on the set of all $\mathrm{P}_{\mathbb{Z}}^{+}$-invariant functions on $\mathscr{B}_{S}^{+}$by

$$
\begin{gathered}
\left.F\right|_{k} \mathrm{P}_{\mathbb{Z}}^{+} g \mathrm{P}_{\mathbb{Z}}^{+}:=\sum_{\imath}\left(\left.F\right|_{k} g_{i}\right) \\
\left(\mathrm{P}_{\mathbb{Z}}^{+} g \mathrm{P}_{\mathbb{Z}}^{+}=\sum_{i} \mathrm{P}_{\mathbb{Z}}^{+} g_{i} \in \mathrm{H}\left(\mathrm{P}_{\mathbb{Z}}^{+}, \mathrm{P}_{\mathbb{Q}}^{+}\right)\right),
\end{gathered}
$$

and we define an embedding of $\mathrm{H}\left(S L_{2}(\mathbb{Z}), M_{2}^{+}(\mathbb{Z})\right.$ into $\mathrm{H}\left(\mathrm{P}_{\mathbb{Z}}^{+}, \mathrm{P}_{\mathbb{\mathbb { Q }}}^{+}\right)$by

$$
p: S L_{2}(\mathbb{Z})\left(\begin{array}{cc}
\alpha & 0 \\
0 & \beta
\end{array}\right) S L_{2}(\mathbb{Z}) \mapsto \mathrm{P}_{\mathbb{Z}}^{+}\left[\left(\begin{array}{cc}
\beta^{-1} & 0 \\
0 & \alpha^{-1}
\end{array}\right)\right] \mathrm{P}_{\mathbb{Z}}^{+},
$$

where $M_{2}^{+}(\mathbb{Z}):=\left\{A \in M_{2}(\mathbb{Z}) \mid \operatorname{det} A>0\right\}$. Let

$$
T_{-}(m):=p\left(\sum_{\substack{\alpha, \beta \in \mathbb{N} \\
\alpha \beta=m, \alpha \mid \beta}} S L_{2}(\mathbb{Z})\left(\begin{array}{ll}
\alpha & 0 \\
0 & \beta
\end{array}\right) S L_{2}(\mathbb{Z})\right)
$$

for any $m \in \mathbb{N}$. Easily we have

$$
T_{-}(m)=\sum_{\substack{a, d \in \mathbb{N} \\
a d=m}} \sum_{b=0}^{d-1} \mathrm{P}_{\mathbb{Z}}^{+}\left[\left(\begin{array}{cc}
d^{-1} & m^{-1} b \\
0 & a^{-1}
\end{array}\right)\right] .
$$

(See Gritsenko [10].)

Proposition 4.1. If $\widetilde{\varphi} \in \mathbb{J}_{k, \tilde{m}}\left(\mathscr{B}_{S}^{+}, \mathrm{P}_{\mathbb{Z}}^{+}\right)$, then $\left.\widetilde{\varphi}\right|_{k} T_{-}(m) \in \mathbb{J}_{k, \tilde{m} m}\left(\mathscr{B}_{S}^{+}, \mathrm{P}_{\mathbb{Z}}^{+}\right)$, where

$$
\left(\left.\widetilde{\varphi}\right|_{k} T_{-}(m)\right)(t, \xi, \tau):=\left\{\left.(\widetilde{\varphi}(t, \xi, \tau) \mathbf{e}(\tilde{m} \omega))\right|_{k} T_{-}(m)\right\} \mathbf{e}(-\widetilde{m} m \omega) .
$$


Proof. Because $T_{-}(m)$ sends the term e $(\tilde{m} \omega)$ to e $(\tilde{m} m \omega),\left.\widetilde{\varphi}\right|_{k} T_{-}(m)$ satisfies the translation formulas of $\mathbb{J}_{k, \tilde{m} m}\left(\mathscr{B}_{S}^{+}, \mathrm{P}_{\mathbb{Z}}^{ \pm}\right)$. Let

$$
\widetilde{\varphi}(t, \xi, \tau)=\sum_{\substack{n \in \mathbb{N} \cup\{0\}, u \in L_{0}^{\prime} \\ 2 n \tilde{m} \geq S_{0}[u]}} A_{(n, u)}(t) \mathbf{e}\left(-{ }^{t} u S_{0} \xi+n \tau\right)
$$

Then we have the Fourier expansion

$$
\left.\widetilde{\varphi}\right|_{k} T_{-}(m)=\sum_{\substack{n \in \mathbb{N} \cup\{0\}, u \in L_{0}^{\prime} a \in \mathbb{N}, a \mid(m, n) \\ 2 n \tilde{m} \geq \geq S_{0}^{0}[u]}} a_{\substack{k-1 \\ a^{-1} u \in L_{0}^{\prime}}} a_{\left(\frac{m n}{a^{2},}, \frac{u}{a}\right)}\left(a^{2} t\right) \mathrm{e}\left(-{ }^{t} u S_{0} \xi+n \tau\right)
$$

by a direct calculation.

Now, let

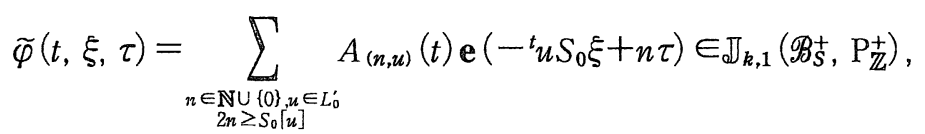

and $A_{(0,0)}(t)=\sum_{n=0}^{\infty} b_{n} t^{n}$. We consider the formal power series

$$
F(Z):=\left(\tilde{A}_{(0,0)}(t)+\sum_{n=1}^{\infty} \sum_{\substack{a \in \mathbb{N} \\ a \mid n}} a^{k-1} A_{(0,0)}\left(a^{2} t\right) \mathbb{e}(n \tau)\right)+\sum_{m=1}^{\infty} \frac{1}{m}\left(\left.\widetilde{\varphi}\right|_{k} T_{-}(m)\right)(t, \xi, \tau) \mathrm{e}(m \omega)
$$

where

$$
\tilde{A}_{(0,0)}(t):=\sum_{n=0}^{\infty} b_{n} \frac{(k+2 n-1) ! \zeta(k+2 n)}{(2 \pi \sqrt{-1})^{k+2 n}} t^{n}
$$

Let

$$
E\left(\widetilde{A}_{(0,0)}\right)(t, \tau):=\frac{1}{2} \sum_{\substack{a b \\
c d \\
c d} \in\left(\begin{array}{c}
1 \\
0 \\
0
\end{array}\right) \backslash S L_{2}(\mathbb{Z})}(c \tau+d)^{-k} \widetilde{A}_{(0,0)}\left(\frac{t}{(c \tau+d)^{2}}\right) .
$$

If the series $\tilde{A}_{(0,0)}(t)$ is a holomorphic function on some domain $D$ and if $k \geq 4$, $E\left(\widetilde{A}_{(0,0)}\right)(t, \tau)$ is a holomorphic function on $D \times \mathbb{H}$.

Lemma 4.2. If $k$ is odd, we have $A_{(0,0)}(t)=0$, hence the first part of $F(Z)$ is zero. If $k$ is even and $k \geq 4, \widetilde{A}_{(0,0)}(t)+\sum_{n=1}^{\infty} \sum_{\substack{a \in \mathbb{N} \\ a \mid n}} a^{k-1} A_{(0,0)}\left(a^{2} t\right)$ e $(n \tau)=E\left(\widetilde{A}_{(0,0)}\right)$ $(t, \tau)$. Hence $F(Z)$ is $\mathrm{P}_{\mathbb{Z}}^{+}$-invariant, formally.

Proof. From the action of $\left[-E_{2}\right]$, we have $\widetilde{\varphi}(t, \xi, \tau)=(-1)^{k} \widetilde{\varphi}(t,-\xi, \tau)$. 
Forms ON THE EXPANDED SyMmETRIC DOMAIN

277

Hence if $k$ is odd, we have $\widetilde{\varphi}(t, 0, \tau)=0$ and $A_{(0,0)}(t)=0$. If $k$ is even,

$$
E\left(\tilde{A}_{(0,0)}\right)(t, \tau)=\tilde{A}_{(0,0)}(t)+\sum_{c=1}^{\infty} \sum_{\substack{d \in \mathbb{Z} \\(d, c)=1}}(c \tau+d)^{-k} \tilde{A}_{(0,0)}\left(\frac{t}{(c \tau+d)^{2}}\right) .
$$

We calculate the Fourier coefficient of it. We have

$$
\begin{aligned}
\int_{0}^{1} \sum_{c=1}^{\infty} \sum_{\substack{d \in \mathbb{Z} \\
(d, c)=1}}(c \tau+d)^{-k} \tilde{A}_{(0,0)}\left(\frac{t}{(c \tau+d)^{2}}\right) \mathbf{e}(-m \tau) d \operatorname{Re}(\tau) \\
=\sum_{c=1}^{\infty} \frac{1}{c}\left\{\sum_{\substack{d \bmod c \\
(d, c)=1}} \mathbf{e}\left(\frac{m d}{c}\right)\right\}\left\{\int_{-\infty}^{\infty} \tau^{-k} \widetilde{A}_{(0,0)}\left(\frac{t}{\tau^{2}}\right) \mathbf{e}\left(-\frac{m \tau}{c}\right) d \operatorname{Re}(\tau)\right\} \\
=\sum_{c=1}^{\infty} \frac{1}{c}\left\{\sum_{\substack{d \bmod c \\
(d, c)=1}} \mathbf{e}\left(\frac{m d}{c}\right)\right\} \\
\quad \times\left\{\sum_{n=0}^{\infty} b_{n} \frac{(k+2 n-1) ! \zeta(k+2 n)}{(2 \pi \sqrt{-1})^{k+2 n}} t^{n}\left\{\int_{-\infty}^{\infty} \tau^{-(k+2 n)} \mathbf{e}\left(-\frac{m \tau}{c}\right) d \operatorname{Re}(\tau)\right\}\right\} \\
=\sum_{n=0}^{\infty} b_{n} t^{n} \zeta(k+2 n)\left\{\sum_{c=1}^{\infty} \frac{m^{k+2 n-1}}{c^{k+2 n}} \sum_{\substack{d \bmod c \\
(d, c)=1}} \mathbf{e}\left(\frac{m d}{c}\right)\right\} \\
=\sum_{n=0}^{\infty} b_{n} t^{n} \sigma_{k+2 n-1}(m) \\
=\sum_{\substack{a \in \mathbb{Z} \\
a \mid m}} a^{k-1} \widetilde{A}_{(0,0)}\left(a^{2} t\right) .
\end{aligned}
$$

This completes the proof.

Lemma 4.3. The formal power series $F(Z)$ is invariant with respect to exchange variables $\omega$ and $\tau$.

Proof. By the equation (8), we have

(9)

$F(Z)=\widetilde{A}_{(0,0)}(t)+\sum_{\substack{n, m \in \mathbb{N} \cup\{0\} \\(n, m) \neq(0,0)}} \sum_{\substack{u \in L_{0}^{\prime} \\ 2 n m} \sum_{0}[u]} \sum_{\substack{a \in \mathbb{N}, a \mid(m, n) \\ a^{-1} u \in L_{0}^{\prime}}} a^{k-1} A\left(\frac{m n}{\left.a^{2}, \frac{u}{a}\right)}\left(a^{2} t\right) \mathbf{e}\left(-{ }^{t} u S_{0} \xi+n \tau+m \omega\right)\right.$.

Let 


$$
\widetilde{F_{\mathbb{Z}}^{+}}:=\left\{\left(\begin{array}{ccc}
1 & 0 & 0 \\
0 & T & 0 \\
0 & 0 & 1
\end{array}\right) \in \Gamma \mid\left(T-E_{l+2}\right) L_{1}^{\prime} \subset L_{1}\right\} .
$$

Lemma 4.4. Formally, $F(Z)$ is $\widetilde{F_{\mathbb{Z}}^{+}}$-invariant.

Proof. By Lemma 2.1 , the equation (9) is $\widetilde{F_{\mathbb{Z}}^{T}}$-invariant.

Fact 4.5. The group, generated by the group $\mathrm{P}_{\mathbb{Z}}^{+}, \widetilde{F_{\mathbb{Z}}^{+}}$and the exchange variables $\omega$ and $\tau$, includes the group

$$
\widetilde{\Gamma}:=\left\{g \in \Gamma \mid\left(g-E_{l+4}\right) L^{\prime} \subset L\right\} .
$$

It is easily induced by Sugano [25, Proposition 6.3].

Remark. If the lattice $L$ is unimodular i.e. $L^{\prime}=L$, then $\widetilde{\Gamma}=\Gamma$.

Proposition 4.6. Formally, $F(Z)$ is $\widetilde{\Gamma}$-invariant.

Proof. It is obvious from previous lemmas.

From now on, we suppose that $\widetilde{\varphi}$ can be written as $\varphi f_{1}$ for some $\varphi \in \mathbb{J}_{k, 1}\left(\mathscr{H}_{S}^{+}\right.$, $\mathrm{P}_{\mathbb{Z}}^{+}$). From Proposition 3.3, we have $b_{n}=0$ for any $n \geq 2$. Hence we have

$$
\tilde{A}_{(0,0)}(t):=\frac{(k-1) ! \zeta(k)}{(2 \pi \sqrt{-1})^{k}} b_{0}+\frac{(k+1) ! \zeta(k+2)}{(2 \pi \sqrt{-1})^{k+2}} b_{1} t
$$

and $E\left(\tilde{A}_{(0,0)}\right)(t, \tau)$ is holomorphic on $\mathbb{C} \times \mathbb{H}$.

Lemma 4.7. If $k \geq 4$ or $A_{(0,0)}(t)=0$, the series $F(Z)$ is a holomorphic function on the domain

$$
\widetilde{\mathscr{B}_{S}^{+}}:=\left\{z=\left(\begin{array}{c}
t \\
w
\end{array}\right) \in \mathscr{B}_{S}^{+}\left|S_{1}[\operatorname{Im}(w)]>\right| t \mid\right\} .
$$

Proof. It is enough to show that

$$
\begin{aligned}
& \sum_{n=1}^{\infty} \frac{1}{m}\left(\left.\widetilde{\varphi}\right|_{k} T_{-}(m)\right)(t, \xi, \tau) \mathbf{e}(m \omega) \\
& =\sum_{m=1}^{\infty}\left\{\frac{1}{m} \sum_{\substack{a, d \in \mathbb{N} \\
a d=m}} \sum_{b=0}^{d-1} a^{k} \widetilde{\varphi}\left(a^{2} t, a \xi, \frac{a \tau+b}{d}\right)\right\} \mathbf{e}(m \omega)
\end{aligned}
$$


is holomorphic on $\widetilde{\mathscr{B}_{S}^{+}}$. To use Hadamard's formula for the radius of convergence with respect to $\mathbf{e}(\omega)$, we estimate

$$
\left|\widetilde{\varphi}\left(a^{2} t, a \xi, \frac{a \tau+b}{d}\right)\right|=\left|f_{1}\left(a^{2} t, \frac{a \tau+b}{d}\right)\right|\left|\varphi\left(a \xi, \frac{a \tau+b}{d}\right)\right| .
$$

First, we estimate $\left|\varphi\left(a \xi, \frac{a \tau+b}{d}\right)\right|$. Let

$$
C:=\sup \left\{|\varphi(\xi, \tau)| \mid \operatorname{Im}(\xi)>0, \operatorname{Im}(\tau) \geq \frac{\sqrt{3}}{2}\right\}
$$

where $\operatorname{Im}(\xi)>0$ means that any component of $\operatorname{Im}(\xi)$ is positive. If $\operatorname{Im}\left(\frac{a \tau+b}{d}\right) \geq$ $\frac{\sqrt{3}}{2}$, we can choose $x \in \mathbb{Z}^{l}$ such that $\operatorname{Im}\left(a \xi+x \frac{a \tau+b}{d}\right)>0$. Then we have

$$
\begin{aligned}
& \left|\varphi\left(a \xi, \frac{a \tau+b}{d}\right)\right| \\
& =\left|\mathbf{e}\left({ }^{t} S_{0} a \xi+\frac{1}{2} S_{0}[x] \frac{a \tau+b}{d}\right)\right|\left|\varphi\left(a \xi+x \frac{a \tau+b}{d}, \frac{a \tau+b}{d}\right)\right| \\
& =\exp \left\{-\pi\left(\frac{a \operatorname{Im}(\tau)}{d} S_{0}\left[x+\frac{d \operatorname{Im}(\xi)}{\operatorname{Im}(\tau)}\right]-\frac{m S_{0}[\operatorname{Im}(\xi)]}{\operatorname{Im}(\tau)}\right)\right\}\left|\varphi\left(a \xi+x \frac{a \tau+b}{d}, \frac{a \tau+b}{d}\right)\right| \\
& \leq C \exp \left(\frac{\pi m}{\operatorname{Im}(\tau)} S_{0}[\operatorname{Im}(\xi)]\right) .
\end{aligned}
$$

If $\operatorname{Im}\left(\frac{a \tau+b}{d}\right)<\frac{\sqrt{3}}{2}$, we can choose $\left(\begin{array}{ll}\alpha & \beta \\ \gamma & \delta\end{array}\right) \in S L_{2}(\mathbb{Z})$ and $p \in \mathbb{Z}$ such that $\operatorname{Im}\left(\frac{\alpha \frac{a \tau+b}{d}+\beta}{\gamma \frac{a \tau+b}{d}+\delta}\right) \geq \frac{\sqrt{3}}{2}$ and $\operatorname{Im}\left(\frac{a \xi+p}{\gamma \frac{a \tau+b}{d}+\delta}\right)>0$. We remark that $\operatorname{Im}\left(\frac{a \tau+b}{d}\right)$ $<\frac{\sqrt{3}}{2}$ induces $\gamma \neq 0$. Then we have

$\left|\varphi\left(a \xi, \frac{a \tau+b}{d}\right)\right|=\left|\gamma \frac{a \tau+b}{d}+\delta\right|^{-k}\left|\mathbf{e}\left(\frac{-\gamma S_{0}[a \xi+p]}{2\left(\gamma \frac{a \tau+b}{d}+\delta\right)}\right)\right| \varphi\left(\frac{a \xi+p}{\gamma \frac{a \tau+b}{d}+\delta}, \frac{\alpha \frac{a \tau+b}{d}+\beta}{\gamma \frac{a \tau+b}{d}+\delta}\right) \mid$.

Because

$$
\left|\gamma \frac{a \tau+b}{d}+\delta\right| \geq \operatorname{Im}\left(\frac{a \tau+b}{d}\right) \geq \frac{\operatorname{Im}(\tau)}{m}
$$

and

$$
\left|\mathbf{e}\left(\frac{-\gamma S_{0}[a \xi+p]}{2\left(\gamma \frac{a \tau+b}{d}+\delta\right)}\right)\right|=\exp \left\{\pi m \operatorname{Im}\left(\frac{S_{0}\left[\xi+\frac{p}{a}\right]}{\tau+\frac{b}{a}+\frac{d \delta}{a \gamma}}\right)\right\} \leq \exp \left(\pi m \frac{S_{0}[\operatorname{Im}(\xi)]}{\operatorname{Im}(\tau)}\right),
$$


We have

$$
\left|\varphi\left(a \xi, \frac{a \tau+b}{d}\right)\right| \leq C\left(\frac{m}{\operatorname{Im}(\tau)}\right)^{k} \exp \left(\frac{\pi m}{\operatorname{Im}(\tau)} S_{0}[\operatorname{Im}(\xi)]\right)
$$

Second, we estimate $\left|f_{1}\left(a^{2} t, \frac{a \tau+b}{d}\right)\right|$. Let

$$
M(T):=\max \left\{\mid \frac{\eta^{\prime}(z)}{\eta(z)} \| \operatorname{Im}(z) \geq T\right\} .
$$

If $\operatorname{Im}(\tau)>T>0$, we have

$$
\left|\frac{d^{j}}{d \tau^{j}} \log \eta(\tau)\right| \leq M(T)(j-1) !(\operatorname{Im}(\tau)-T)^{-j} .
$$

Hence we have

$$
\left|f_{1}(t, \tau)\right| \leq 1+\sum_{j=1}^{\infty} \frac{2 M(T)}{j !}\left(\frac{\pi|t|}{\operatorname{Im}(\tau)-T}\right)^{j}=1+2 M(T)\left(e^{\frac{\pi|t|}{\operatorname{Im}(\tau)-T}}-1\right) .
$$

By the equation (5), we have

$$
\begin{aligned}
M(T) & \leq \frac{\pi}{12}+2 \pi \sum_{n=1}^{\infty} \frac{n(n+1)}{2}\left(e^{-2 \pi T}\right)^{n} \\
& <2 \pi \sum_{n=0}^{\infty} \frac{(n+1)(n+2)}{2}\left(e^{-2 \pi T)^{n}}\right. \\
& =\frac{2 \pi}{\left(1-e^{-2 \pi T}\right)^{3}}
\end{aligned}
$$

Let $T=\frac{a \epsilon}{d} \operatorname{Im}(\tau)$ for $0<\epsilon<1$. Because $T \geq \frac{\epsilon}{m} \operatorname{Im}(\tau)$, we have $M(T) \leq$ $M\left(\frac{\epsilon}{m} \operatorname{Im}(\tau)\right)$. Hence we have

$$
\begin{aligned}
\left|f_{1}\left(a^{2} t, \frac{a \tau+b}{d}\right)\right| & \leq 1+2 M\left(\frac{\epsilon}{m} \operatorname{Im}(\tau)\right)\left(e^{\frac{m \pi|t|}{(1-\epsilon) \operatorname{Im}(\tau)}}-1\right) \\
& <1+\frac{4 \pi}{\left(1-e^{-\frac{2 \pi \epsilon \operatorname{Im}(\tau)}{m}}\right)^{3}}\left(e^{\frac{m \pi|t|}{(1-\epsilon \operatorname{Im}(H \tau)}}-1\right)
\end{aligned}
$$

and

(10)

$$
\begin{aligned}
\left|\frac{1}{m}\left(\left.\widetilde{\varphi}\right|_{k} T_{-}(m)\right)(t, \xi, \tau)\right|< & C m^{k+2}\left\{\max \left(1, \frac{m}{\operatorname{Im}(\tau)}\right)\right\}^{k} \exp \left(\frac{\pi m}{\operatorname{Im}(\tau)} S_{0}[\operatorname{Im}(\xi)]\right) \\
& \times\left\{\frac{4 \pi}{\left(1-e^{-\frac{2 \pi \epsilon \operatorname{Im}(\tau)}{m}}\right)^{3}}\left(e^{\frac{m \pi|t|}{(1-\epsilon) \operatorname{Im}(\tau)}}-1\right)\right\}
\end{aligned}
$$


for any $0<\epsilon<1$. Therefore we have

$$
\limsup _{m \rightarrow \infty}\left|\frac{1}{m}\left(\left.\widetilde{\varphi}\right|_{k} T_{-}(m)\right)(t, \xi, \tau)\right|^{\frac{1}{m}} \leq \exp \left\{\frac{\pi S_{0}[\operatorname{Im}(\xi)]}{\operatorname{Im}(\tau)}+\frac{\pi|t|}{(1-\epsilon) \operatorname{Im}(\tau)}\right\}
$$

for any $0<\epsilon<1$, that is,

$$
\limsup _{m \rightarrow \infty}\left|\frac{1}{m}\left(\left.\widetilde{\varphi}\right|_{k} T_{-}(m)\right)(t, \xi, \tau)\right|^{\frac{1}{m}} \leq \exp \left\{\frac{\pi\left(S_{0}[\operatorname{Im}(\xi)]+|t|\right)}{\operatorname{Im}(\tau)}\right\} .
$$

Because the right hand of $(10)$ is monotone decreasing with respect to $\operatorname{Im}(\tau)$ and monotone increasing with respect to $S_{0}[\operatorname{Im}(\xi)]$ and $|t|$, the series $F(Z)$ converges absolutely and locally uniformly on $\widetilde{\mathscr{B}_{S}^{+}}$.

Corollary 4.8. If $k \geq 4$ or $A_{(0,0)}(t)=0, F(Z)$ is a holomorphic $\widetilde{\Gamma}$-invariant function on $\widetilde{\mathscr{B}_{S}^{+}}$.

Proof. It is obvious from Proposition 4.6 and Lemma 4.7.

Lemma 4.9. The domain $\widetilde{\mathscr{B}_{S}^{+}}$contains the fundamental domain of $\mathscr{B}_{S}^{+}$with respect to the group $\mathrm{P}_{\mathbb{Z}}^{+}$. Hence we have an open covering

$$
\mathscr{B}_{S}^{+}=\bigcup_{r \in \Gamma} r \widetilde{\mathscr{B}_{S}^{+}} .
$$

Proof. By the equation (1), the condition of $\widetilde{\mathscr{B}_{S}^{+}}$is

$$
\left(\frac{1}{\operatorname{inv}(Z)}-2\right)|t|>\operatorname{Re}(t) \Leftrightarrow \frac{1}{\operatorname{inv}(Z)}-2>\cos (\arg t) .
$$

Let $A=\left(\begin{array}{ll}a & b \\ c & d\end{array}\right) \in S L_{2}(\mathbb{Z})$, then $[A]$ maps $t$ to $\frac{t}{(c \tau+d)^{2}}$. Let $Z \in \mathscr{B}_{S}^{+}$. Because $\operatorname{Im}(\tau) \neq 0$, we can choose $A$ such that

$$
\frac{1}{\operatorname{inv}(Z)}-2>\cos \left(\arg \frac{t}{(c \tau+d)^{2}}\right)
$$

Hence $[A] Z=\widetilde{\mathscr{B}_{S}^{+}}$. This completes the proof.

By Corollary 4.8 and Lemma 4.9 , we can define $F(Z):=J(g, \widetilde{Z})^{k} F(\widetilde{Z})$ for any $Z$ $\in \mathscr{B}_{S}^{+}$, where $Z=g \widetilde{Z}, g \in \widetilde{\Gamma}$ and $\widetilde{Z} \in \widetilde{B_{S}^{+}}$. This definition does not depend on the choice of $g$ and $\widetilde{Z}$, because $F(Z)$ is $\widetilde{\Gamma}$-invariant on $\widetilde{\mathscr{B}_{S}^{+}}$.

Now, we have our main theorem. 
Theorem 4.10. If $k \geq 4$ or if $A_{(0,0)}(t)=0, F(Z)$ is a holomorphic $\widetilde{\Gamma}$-invariant function on $\mathscr{B}_{S}^{+}$. Moreover, if the lattice $L$ is unimodular, then $F(Z) \in \mathbb{M}_{k}\left(\mathscr{B}_{S}^{+}, \Gamma\right)$.

Proof. It is obvious from the definition of $F(Z)$ on $\mathscr{B}_{S}^{+}$.

Remark. Now we have the following commutative diagram.

$$
\begin{array}{ccc}
\mathbb{J}_{k, 1}\left(\mathscr{H}_{S}^{+}, \mathrm{P}_{\mathbb{Z}}^{+}\right) \ni \varphi & \multirow{\text{Gritsenko}[10,11]}{\longrightarrow}{} & * \in \mathbb{M}_{k}\left(\mathscr{H}_{S}^{+}, \widetilde{\Gamma}\right) \\
\times f_{1} \downarrow & \circlearrowleft & \\
& \circlearrowleft \uparrow & \\
\mathbb{J}_{k, 1}\left(\mathscr{B}_{S}^{+}, \mathrm{P}_{\mathbb{Z}}^{+}\right) \ni \widetilde{\varphi}=\varphi \times f_{1} & \stackrel{\text { Theorem } 4.10}{\longrightarrow} & F(Z) \in \mathbb{M}_{k}\left(\mathscr{B}_{S}^{+}, \widetilde{\Gamma}\right)
\end{array}
$$

\section{Acknowledgment}

I thank Professor Kyoji Saito and Doctor Yoshiki Hayashi for useful advice and discussions. I thank Professor Valeri A. Gritsenko for his instructive lectures at RIMS in the fall of 1996. I thank Professor Takashi Sugano, Professor Masaru Ueda and Professor Atsushi Murase for useful advice at the fifth summer school of number theory in 1997.

\section{References}

[1] Ahlfors, L. V., Complex Analysis, Third Edition, McGraw-Hill, 1966, 1979.

[2] Arnold, V. I., Classification of Unimodular Critical Points of Functions, Functional Anal. Appl., 7 (1973), 230-231.

[3] Baily, W. L., Introductory Lectures on Automorphic Forms, Princeton-Univ. Press, IwanamiSyoten, Tokyo, 1973.

[4] Borcherds, R. E., Automorphic Forms on $O_{s+2,2}(\mathbf{R})$ and Infinite Products, Inv. Math., 120 (1995), 161-213.

[5] Brieskorn, E., The Unfolding of Exceptional Singularities, Nova Acta Leopoldina, NF $52 \mathrm{Nr}$. 240 (1981), 65-93.

[6] Dolgacev, I. V. and Nikulin, V. V., The Exceptional Singularities of V. I. Arnol'd, and $K 3$ Surfaces (Russian), Seventh All-Union Topology Conf. (Minsk, 1977), Abstracts of Lectures and Communications, Inst. Mat. Akad. Nauk BSSR, Minsk, 1977.

[7] Eguchi, T., Topological Field Theory to Tokuiten Riron (Japanese), Seminar note, 1993.

[8] Eichler, M. and Zagier, D., The Theory of Jacobi Forms, Progr. Math., Birkhäuser, Boston, 55 (1985)

[9] Freitag, E., Siegelsche Modulfunktionen (German), GMW254, Springer Verlag, Berlin, 1983.

[10] Gritsenko, V. A., Fourier-Jacobi Functions of $n$ Variables, Zap. Nauk. Sem. LOMI, 168 (1988), 32-45; English transl. in J. Soviet Math., 53 (1991), 243-252.

[11] _ Modular Forms and Moduli Spaces of Abelian and K3 Surfaces, Algebra i Analyz, $6: 6$ (1994), 65-102; English transl. in St. Petersburg Math. J., 6:6 (1995), 1179-1208.

[12] Kawai, T., $n=2$ Heterotic String Threshold Correction, $K 3$ Surface and Generalized 
Kac-Moody Superalgebra, hep-th 9512046, 1995.

[13] Looijenga, E., The Smoothing Components of a Triangle Singularity. I, Proc. Sympos. Pure Math., 40-2 (1983), 173-183.

[14] _ . The Smoothing Components of a Triangle Singularity. II , Math. Ann., 269 (1984), 357-387.

[15] Oda, Takayuki, On Modular Forms Associated with Infinite Quadratic Forms of Signature (2, n+2), Math. Ann., 231 (1977), 97-144.

[16] Oda, Tadao, K. Saito's Period Mapping for Holomorphic Functions with Isolated Critical Points, Adv. Stud. Pure Math., 10 (1987) (Alg. Geom. Sendai 1985), 591-648.

[17] Pinkham, H. C., Singularités cxceptionelles, la dualité étrange d'Arnold et les surfaces $K 3$ (French), C. R. Acad. Sc. Paris, 284 (1977), 615-618.

[18] Pyatetski-Shapiro, I. I., Automorphic Functions and the Geometry of Classical Domains, Gordon and Breach, New York, 1969.

[19] Saito, K., Period Mapping Associated to a Primitive Form, Publ. RIMS, Kyoto Univ., 19 (1983), 1231-1264.

[20]__ Futei Nijikeishiki $I$ ni Taiousuru Syukisyazo $B_{v}$ (Japanese), Seminar memo, 1991.

[21] _ Around the Theory of the Generalized Weight System: Relations with Singularity Theory, the Generalized Weyl Group and Its Invariant Theory, Etc. Amer. Math. Soc. Transl., 183-2 (1998), 101-143.

[22] _ Period Domain for Primitive Forms of Witt Index $\leq 2$, In preparation.

[23] Siegl, C. L., Topics in complex function theory, I , II , III, Wiley-Interscience, 1969, 1971, 1973.

[24] Satake, I., Flat structure and the prepotential for the elliptic root system of type $D_{4}^{(1,1)}$, Topological Field Theory, Primitive Form and Related Topics. Birkháuser, 1998.

[25] Sugano, T., Jacobi Forms and the Theta Lifting, Comment. Math. Univ. St. Paul., 44-1 (1995), $1-58$.

[26] Zagier, D., Sur la conjecture de Saito-Kurokawa (d'apres H. Maass) Seminaire DelangePisot-Poitou 1979-1980, Progr. Math., 5 (1980), 371-394. 
